\title{
INVESTIGACIONES
}





\title{
Verdad y corrección en la interpretación jurídica*
}

\author{
Lorena Ramírez Ludeña**
}

\begin{abstract}
RESUMEN
En este trabajo cuestiono que el valor de verdad de los enunciados interpretativos constituya un candidato idóneo para diferenciar las teorías acerca de la interpretación jurídica. Las concepciones acerca de la interpretación por parte de los jueces suelen ser diferenciadas en función de la posición que asumen respecto del valor de verdad de los enunciados interpretativos. Analizaré varios argumentos para defender y rechazar que los enunciados interpretativos tienen valor de verdad, tomando especialmente en cuenta los argumentos que el realismo moderado ha dirigido contra el formalismo moderado. Concluiré que es mejor hacer referencia a la corrección de las interpretaciones en lugar de al valor de verdad de los enunciados interpretativos.
\end{abstract}

Interpretación jurídica - instrumentos interpretativos - enunciados interpretativos valor de verdad - corrección

\section{Truth and correction in legal interpretation}

\begin{abstract}
In this paper I call into question that the truth value of interpretative statements constitutes a plausible basis for distinguishing theories on legal interpretation. The conceptions about judicial interpretation are usually differentiated in virtue of the position they assume regarding the truth value of interpretative statements. I will analyze some arguments to defend, and some others to reject, that interpretative statements have truth value, taking mainly into account the criticism that moderate realism has addressed to moderate formalism. I will conclude that it is better to make reference to the correction of the interpretations, instead of the truth value of interpretive statements.
\end{abstract}

Legal interpretation - interpretative arguments - interpretative statements truth value - correction

* Agradezco a Josep María Vilajosana, Diego Papayannis, José Juan Moreso, Sebastián Agüero, Alberto Carrió, Jorge Rodríguez, Claudina Orunesu, y a todos los miembros del grupo de investigación de teoría del derecho de la Universidad de Mar del Plata, sus comentarios a una versión previa de este trabajo. Algunas de las ideas que aquí se desarrollan fueron presentadas en el XIX Seminario ítalo-español-francés de teoría del derecho, cuyas ponencias han aparecido publicadas en la revista Analisi e Diritto. Agradezco también a los participantes del seminario sus críticas y sugerencias. Finalmente, agradezco las sugerencias de los árbitros de la revista.

** Profesora de la Universidad Pompeu Fabra, Barcelona. Correo electrónico: lorena.ramirez@upf.edu. Artículo recibido el 2 de octubre de 2014 y aceptado para su publicación el 27 de marzo de 2015. 


\section{INTRODUCCIÓN}

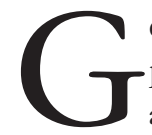

eneralmente cuando se analizan las diversas teorías acerca de la interpretación por parte de los jueces se hace referencia a dos concepciones radicales. Así, aunque no sea tarea sencilla encontrar a sus partidarios, es habitual diferenciar dos posiciones extremas: el realismo y el formalismo radical. Por un lado, suele afirmarse que, desde una perspectiva realista radical, los enunciados interpretativos nunca son verdaderos o falsos. El derecho depende de lo que decidan los jueces y no es posible sostener que se equivocan al interpretar las disposiciones jurídicas. Por otro lado, desde una concepción formalista radical, los enunciados interpretativos son siempre verdaderos o falsos y los jueces sí pueden equivocarse en sus interpretaciones ${ }^{1}$.

En la literatura iusfilosófica se han defendido posiciones menos radicales, en apariencia mucho más plausibles.

Pese a sostener, del mismo modo que el realismo radical, que los enunciados interpretativos carecen de valor de verdad, se ha argumentado que los jueces crean derecho en lugar de interpretarlo si optan por interpretaciones que se hallan fuera del marco de interpretaciones admisibles en la comunidad. De acuerdo con esta posición, los jueces cuentan con diferentes instrumentos interpretativos, que conducen a diferentes soluciones, entre los que pueden optar. Entonces, en un sentido relevante, el derecho es lo que los jueces dicen que es. Pero hay límites a lo que pueden hacer en tanto intérpretes. Llamaré a esta posición "realismo moderado" 2 .

Además, se ha defendido una posición moderada por parte de quienes entienden que los enunciados interpretativos sí tienen valor de verdad en los casos claros, aunque también

\footnotetext{
${ }^{1}$ Como ha señalado Barberis (2000), no resulta fácil determinar qué rasgos diferencian a las diversas concepciones interpretativas, pero espero que esta caracterización básica resulte suficientemente intuitiva y que sea además de utilidad para reflexionar acerca de cuál es el elemento central en la discusión entre los diferentes autores. Respecto del escepticismo extremo, Ruiz Manero (2012: 204 y ss.) ha criticado que sea una concepción propiamente interpretativa, en disputa con el resto, precisamente porque puede afirmarse que no hace referencia a una genuina interpretación de textos jurídicos.

${ }^{2}$ Esta es la posición de Riccardo Guastini. Véase, por ejemplo, Guastini 2012, donde señala que en los sistemas jurídicos existen diversos instrumentos interpretativos, que son además cambiantes. Los diferentes instrumentos suponen que las disposiciones no expresan una única norma, sino una multiplicidad de ellas entre las que el intérprete puede optar, porque no existen metacriterios para seleccionar una interpretación. Quien interpreta adscribe un significado a un texto, entre otros posibles, por lo que los enunciados interpretativos carecen de valor de verdad. Aunque en este trabajo adoptaré como posición de referencia la concepción de Guastini, esta no es, evidentemente, la única alternativa realista moderada, ni tampoco la única alternativa realista a la genovesa. Véase Chiassoni (1998), quien analiza elementos que suelen considerarse propios del realismo genovés, como el carácter estipulativo y prescriptivo de la interpretación jurídica que, como veremos, son cuestionados por Guastini. Véase también Ferrer-Ratti 2011. Existen al menos tres estrategias para fundamentar una concepción realista: ser escéptico acerca de los significados en general (véase en este sentido, Kripke, 1982); ser escéptico, como en el caso de Tarello (1974), acerca de la interpretación de las prescripciones jurídicas; o, como sucede con Guastini (2012), destacar la existencia de numerosos instrumentos interpretativos. Esta última estrategia parece ser la única de ellas que permite articular una concepción realista moderada.
} 
sostienen que hay casos difíciles en que carecen de valor de verdad. Denominaré a esta concepción "formalismo moderado"3.

Como acabo de señalar, un elemento central a efectos de diferenciar las diversas posiciones parece ser si estas sostienen que tiene sentido hablar o no de la verdad de los enunciados interpretativos, y, si consideran que sí, en qué casos. Precisamente por ello, los enunciados interpretativos constituirán el principal elemento de análisis en este trabajo. Los enunciados interpretativos, generalmente implícitos en las decisiones judiciales tienen la forma " $X$ significa $Y$ " . En este esquema, " $X$ " e " $Y$ " son variables metalingüísticas que están en lugar del nombre del enunciado interpretado y del enunciado interpretante, respectivamente. El juez menciona ambos enunciados para afirmar la existencia de una relación de sinonimia entre ambos.

En este trabajo trataré, en primer lugar, de determinar qué es lo que explica que una concepción realista moderada rechace la posibilidad de que los enunciados interpretativos sean verdaderos o falsos. Analizaré además si alguna de esas explicaciones es suficiente para fundamentar que se niegue que los enunciados interpretativos tienen valor de verdad. Cuestionaré finalmente que el valor de verdad de los enunciados interpretativos sea un elemento determinante a efectos de caracterizar las diferentes concepciones acerca de la interpretación jurídica. En la literatura acerca de la cuestión, el realismo extremo, el formalismo extremo y el formalismo moderado, son denominados, respectivamente, "la pesadilla", "el noble sueño" y "la vigilia", denominaciones que también emplearé en ocasiones a lo largo de este trabajo ${ }^{5}$. Como he señalado, estas posiciones suelen ser caracterizadas en virtud de su posición respecto de la verdad de los enunciados interpretativos, pero también, frecuentemente de manera indistinta, en función de si aceptan o no que hay interpretaciones correctas. En la última parte del trabajo distinguiré ambos aspectos y argumentaré a favor de que el criterio determinante para caracterizar las distintas posiciones sea la corrección de las interpretaciones y no la verdad de los enunciados interpretativos.

\section{LOS ARGUMENTOS DEL REALISMO MODERADO}

Como he señalado en la introducción, pese a sostener que puede diferenciarse la interpretación de la creación del derecho, el realismo moderado rechaza que tenga sentido hacer

\footnotetext{
3 Adopto esta terminología a efectos expositivos, con el propósito de poner en relación las distintas concepciones. En este sentido, aunque puede resultar extraño considerar que Hart sostuvo algún tipo de formalismo, su posición en Hart (1994) quedaría incluida, de acuerdo con lo aquí expuesto, entre los formalistas moderados. En todo caso, si tenemos en cuenta que en el esquema hartiano los casos difíciles son vistos como marginales, la caracterización de su posición como una forma moderada de formalismo no parece tan desencaminada.

${ }^{4}$ Aunque emplee esta forma abreviada a efectos expositivos, creo, siguiendo a Hernández Marín (2008), que los enunciados interpretativos tienen la forma " $X$ significa lo mismo que $Y$ ". Para Guastini, uno de los autores con mayor incidencia en el debate, los enunciados interpretativos tienen la forma "“ $\mathrm{X}$ " significa " $\mathrm{Y}$ ”". No obstante, no creo que esta diferencia afecte al análisis que se realizará a continuación.

${ }^{5}$ Véase Moreso 1997: 186.
} 
referencia al valor de verdad de los enunciados interpretativos. Pero si bien es cierto que no es fácil encontrar en la literatura argumentos a favor de considerar que algunos enunciados interpretativos tienen valor de verdad, tampoco lo es, sorprendentemente, hallar una explicación clara de por qué, para el realista moderado, ello no sería así. A continuación reconstruiré y criticaré diferentes argumentos que pueden ofrecerse desde el realismo moderado para rechazar que los enunciados interpretativos tienen valor de verdad. Con frecuencia, los realistas han planteado estos argumentos en su debate con los formalistas moderados, partidarios de la vigilia. Diferenciar los argumentos y analizarlos por separado permitirá apreciar mejor si son o no concluyentes ${ }^{6}$.

\section{Problemas relativos al lenguaje ordinario}

\subsection{Los casos fáciles}

En ocasiones se ha señalado que el modelo formalista moderado confiere al lenguaje natural un rol en la interpretación del derecho que en realidad no tiene. Como es sabido, Hart destacó la relevancia del lenguaje ordinario (y de sus problemas) en relación con la guía de conducta en el ámbito jurídico. De acuerdo con los realistas moderados, esa podría ser la explicación de por qué Hart entiende que hay enunciados interpretativos verdaderos y falsos: porque sostiene que hay un significado prejurídico que es claro, y que determina la verdad de los enunciados interpretativos en los casos que no plantean dificultades. Por tanto, en los casos claros los enunciados interpretativos serían verdaderos por la incidencia que en el derecho tiene el significado ordinario. En otras palabras, de acuerdo con la vigilia, los términos tendrían un significado propio, independiente de consideraciones jurídicas. En cambio, según los realistas, las particularidades del ámbito jurídico conllevan que el lenguaje

\footnotetext{
${ }^{6}$ En mi exposición me centraré, fundamentalmente, en Guastini 1999, 2008, 2010 y 2012. De acuerdo con Guastini (2012: 31 y ss.), puede distinguirse la interpretación cognitiva, en que se describen las diversas opciones interpretativas, de la decisoria, en que se elige una de esas opciones. La interpretación creativa supone asumir una interpretación fuera del marco de opciones interpretativas según la interpretación cognitiva. Los enunciados interpretativos serían aquellos mediante los cuales los jueces escogen una de las interpretaciones posibles. El esquema guastiniano es problemático, ya que, como señala Lifante (2012), no resulta claro qué debe entenderse por interpretación cognitiva: o bien es una actividad descriptiva, que consiste en la descripción de la interpretación efectivamente hecha por otros, pero en este caso no es realmente interpretación, o bien es una identificación de significados efectivos y posibles, y en este sentido es interpretación genuina, pero entonces no es mera descripción. En este sentido, creo que no queda claro si la interpretación cognitiva consiste, de acuerdo con Guastini, en la descripción de los significados empleados efectivamente en la comunidad jurídica, en los significados posibles, o en los significados que es previsible que sean considerados relevantes. Lifante también critica la distinción entre interpretación cognitiva, decisoria y creativa. Si la interpretación decisoria es aquella que se produce eligiendo entre los significados identificados mediante la interpretación cognitiva y la interpretación creativa es aquella que se produce asociando a la disposición un significado por fuera de aquellos identificados por la interpretación cognitiva, entonces la distinción es problemática si se afirma además que la interpretación cognitiva consiste en identificar los significados que los jueces han decidido asociar a los textos. En lo que aquí interesa, ello puede resultar problemático si conlleva la contraintuitiva conclusión de que los jueces no emplean genuinos enunciados interpretativos hasta que no se ha desarrollado una práctica interpretativa y existen diversas opciones entre las que escoger.
} 
natural no determine el significado de las disposiciones, por lo que la verdad de los enunciados interpretativos no puede fundamentarse apelando al significado propio de los términos ${ }^{7}$.

La crítica anterior por parte del realismo moderado agrupa en realidad tres críticas que están relacionadas entre sí. Por un lado, que los formalistas moderados entienden que las palabras tienen un significado propio. Por otro, que ese significado propio, que viene dado por los lenguajes naturales, es independiente de consideraciones jurídicas. En tercer lugar, que ese significado es el único admisible para el formalista moderado. Por mi parte, considero que la vigilia no tiene que asumir lo que el realista moderado le atribuye. A continuación distinguiré y analizaré los tres argumentos por separado.

En primer lugar, quienes sostienen que, al menos en algunos casos, hay respuestas correctas, no tienen por qué comprometerse con que las palabras tienen un significado propio, que es independiente de la conducta, las creencias y las actitudes de los individuos. Aunque me centraré en este punto con posterioridad, cuando analice la naturaleza adscriptiva de la interpretación, es importante anticipar que creo que la vigilia puede asumir que en los casos fáciles comprendemos y en los difíciles interpretamos, pero también que siempre se produce una interpretación de los textos jurídicos ${ }^{8}$. Ello en ningún caso supone comprometerse con que el lenguaje no es arbitrario: los partidarios de la vigilia entienden que el lenguaje es una creación humana, que el vínculo entre las palabras, los significados, y aquello a lo que referimos es arbitrario, lo que impide que se los pueda criticar porque defienden la relevancia de algo así como un significado propio de las palabras.

Tampoco parece ser necesario que se comprometan con que las disposiciones tienen un significado dependiente de los lenguajes naturales, prescindiendo de las particularidades de la interpretación jurídica. De hecho, cuando defienden que el lenguaje ordinario es relevante, pueden también sostener que lo es en atención a las particularidades del ámbito jurídico. Por ejemplo, si a efectos de destacar la importancia del lenguaje ordinario señalan la relevancia que en el derecho tiene la guía de conducta.

Pero, además, no tienen que comprometerse con que siempre el lenguaje ordinario es determinante. En este sentido, los defensores de la vigilia pueden admitir que un supuesto sea considerado fácil en atención, por ejemplo, a la intención del legislador ${ }^{9}$.

7 Para Guastini (2008: 13 y 14) no hay un significado propio que venga dado por el significado ordinario o por las intenciones. De acuerdo con su posición, las teorías que señalan la relevancia de dichas interpretaciones en el ámbito jurídico constituyen en realidad teorías normativas de la interpretación.

${ }^{8}$ En este trabajo entenderé que, ya se trate de un caso fácil o difícil, ya se entienda o no que lo que ocurre en los casos fáciles es diferente de lo que ocurre en los difíciles, el juez profiere o asume un enunciado interpretativo en tanto relaciona los términos o las disposiciones con un significado. Estoy empleando aquí el término "interpretación" en dos sentidos distintos, en un caso como algo distinto de la comprensión, y (en el sentido que adopto en el texto) como algo que abarca la compresión. Si entendiéramos en cambio que la vigilia sostiene que en los casos claros no se interpreta tampoco en el sentido amplio al que me refiero en esta nota, y que por tanto el juez no profiere o asume enunciados interpretativos en tales casos, el debate con el realismo moderado carecería de sentido: ambas teorías sostendrían que los enunciados interpretativos carecen de valor de verdad.

${ }^{9}$ Probablemente, la explicación más plausible de lo que ocurre en los casos fáciles sea que captamos el significado de las disposiciones, aunque estas se expresan haciendo uso de lenguaje ordinario, y que dicho significado no puede ser problematizado en atención a otros instrumentos interpretativos, precisamente porque 


\subsubsection{Los casos difíciles}

La diferencia entre el realismo moderado y la vigilia en relación con los enunciados interpretativos quizá esté, no en cómo la vigilia ve los casos fáciles, sino en cómo reconstruye los casos difíciles. De acuerdo con la concepción realista moderada, la vigilia sostiene que los casos difíciles vienen dados por problemas relativos a los límites del lenguaje ordinario, desconociendo así la incidencia de los diferentes instrumentos interpretativos, característicamente jurídicos. Pero si bien es cierto que en un primer momento Hart destacó los problemas de vaguedad y textura abierta de los conceptos de clase, posteriormente señaló la incidencia de los distintos instrumentos interpretativos, que no solo tienen un impacto en la consideración de un caso como difícil, sino que además constituyen el instrumento idóneo para la resolución de la cuestión jurídica de un modo no arbitrario ${ }^{10}$. Entonces, si lo que señala Hart es plausible, no parece que los partidarios de la vigilia tengan que comprometerse con que los casos difíciles son únicamente casos marginales fruto de los límites del lenguaje ordinario. Y, en lo que aquí interesa, su aceptación de que los enunciados interpretativos tienen valor de verdad no se basa en su desconocimiento del impacto de los instrumentos interpretativos.

Otro modo de plantear esta cuestión consiste en señalar que la vigilia solo presta atención al fenómeno de la subsunción, y no propiamente a la interpretación en abstracto. En este sentido, una vez que asume en el nivel de la interpretación en abstracto la relevancia del lenguaje ordinario, se centra en los problemas subsuntivos. Así, podría sostenerse por parte del realismo moderado que los problemas en los que se centra la concepción formalista moderada son problemas de vaguedad y textura abierta, que tienen que ver con la subsunción de casos cuando ya se ha asumido una determinada interpretación, y no propiamente con la interpretación en abstracto, y que solo esta última es determinante a efectos de hacer referencia al valor de verdad de los enunciados interpretativos ${ }^{11}$. Entonces, el formalismo moderado creería erróneamente que hay enunciados interpretativos que tienen valor de verdad, en atención al lenguaje ordinario.

todos ellos resuelven un gran número de supuestos del mismo modo (Moreso 1997: 222). En este sentido, me parece difícil negar que el lenguaje ordinario desempeña un rol fundamental en nuestra comprensión de las disposiciones jurídicas. Pero ello no puede conducirnos a afirmar que, de acuerdo con la vigilia, solo el lenguaje ordinario es relevante.

${ }^{10}$ Hart 1980: 6. Con algunas diferencias, presenté este argumento y el anterior en Ramírez Ludeña 2012: 92 y ss.

${ }^{11}$ Guastini (2010: 126 y ss.) señala que la vigilia solo se refiere a enunciados subsuntivos, y que acordaría con el formalismo radical en que los enunciados interpretativos son verdaderos o falsos. De este modo, la vigilia no representaría una posición distinta del formalismo radical por lo que respecta a los enunciados interpretativos. Entonces, el realismo moderado y la vigilia no tendrían un genuino desacuerdo sobre los enunciados interpretativos (aunque, obviamente, sí lo harían de modo indirecto), ya que el realismo moderado se refiere a los enunciados interpretativos, pero la vigilia a los subsuntivos. 
Guastini no parece encontrar problemático el predicar verdad o falsedad de los enunciados subsuntivos, pero rechaza que ello sea así en el caso de los enunciados interpretativos ${ }^{12}$. La cuestión central es entonces qué diferencia los enunciados subsuntivos de los interpretativos como para poder afirmar que unos sí pueden ser verdaderos o falsos, pero no los otros. Según Guastini, aunque en la actividad judicial pueda resultar difícil distinguir la interpretación en abstracto (que Guastini vincula con problemas de equivocidad) y la orientada a hechos (relacionada con problemas de vaguedad), ambos procesos son lógicamente independientes: atribuir significado a un texto es algo previo y distinto, aunque relacionado con la clasificación de casos. Sin embargo, ya sea que Guastini se refiera a la subsunción individual o a la genérica ${ }^{13}$, considero que la interpretación orientada a hechos no parece representar un problema interpretativo distinto de la interpretación de textos. Es cierto que ambas actividades son separables y que la interpretación en abstracto es (conceptualmente) previa. Pero Guastini no parece tomarse suficientemente en serio la incidencia de la interpretación en abstracto en la interpretación orientada a hechos. Si asumimos que el sentido de una palabra, expresión u oración es claro, y además conocemos los diferentes aspectos del caso particular -lo que tiene que ver con la prueba y no propiamente con la interpretación- no resta por llevar a cabo ninguna actividad interpretativa adicional. Por ejemplo, si al interpretar el término "llave" establecemos que un determinado rasgo (que incluye a las tarjetas magnéticas) es fundamental, no habrá ninguna otra actividad interpretativa adicional a llevar a cabo para considerar la inclusión de las tarjetas magnéticas en general o la inclusión de una determinada tarjeta magnética en el ámbito de aplicación de la norma. Y ello pese a que, evidentemente, calificar un caso concreto como una instancia de "llave" constituye una actividad distinta de interpretar en abstracto el término "llave". Los problemas que se plantean a partir de casos como el de las tarjetas magnéticas no son problemas interpretativos distintos de los señalados por Guastini en relación con la existencia de numerosas interpretaciones: las tarjetas magnéticas nos plantean problemas precisamente porque, por ejemplo, tenemos dudas respecto de si lo relevante es el lenguaje ordinario o la intención del legislador. Entonces, si no existe un argumento en la posición de Guastini que fundamente la diferencia en el plano interpretativo entre enunciados interpretativos y subsuntivos, la posibilidad de predicar la verdad de algunos enunciados subsuntivos -que es admitida por Guastini- nos conduciría, salvo que se señale una diferencia relevante, también a admitir la posibilidad de predicar la verdad de determinados enunciados interpretativos ${ }^{14}$.

12 Así, Guastini (2010: 126, n. 15) cree que hay enunciados subsuntivos verdaderos bajo la condición de que "el supuesto de hecho concreto sea descrito con los mismos términos utilizados en la formulación (o en la reformulación interpretativa) de la norma”.

${ }^{13}$ Los ejemplos de Guastini relativos a la interpretación orientada a hechos, como es el caso de si las bicicletas son vehículos, parecen indicar que está haciendo referencia a la subsunción genérica y no a la subsunción individual, de casos situados espacio-temporalmente.

${ }^{14}$ Esto mismo ha sido sostenido por Ruiz Manero 2012: 216. Si atendemos a los ejemplos introducidos por Guastini, parece que los diferentes supuestos pueden ser reconstruidos indistintamente como problemas interpretativos relativos a enunciados o a hechos. Así, el caso de las bicicletas, que Guastini emplea como un claro ejemplo de subsunción, puede ser reconstruido como un supuesto en que no sabemos si el enunciado "prohibida la entrada de vehículos en el parque" expresa la norma relativa a la prohibición de cualquier 


\subsection{El número de casos controvertidos}

Si la cuestión central no es simplemente la incidencia de los instrumentos interpretativos, aunque las distintas posiciones reconocen su relevancia, quizá lo sea el número de casos en que, a tenor de cada una de las concepciones, dichos instrumentos son importantes. En este sentido, la concepción formalista moderada consideraría menor la incidencia de los instrumentos, y los casos problemáticos en términos interpretativos serían residuales. El realismo moderado, en cambio, enfatiza que la existencia de numerosos instrumentos interpretativos conduce a que el juez cuente con diversas opciones interpretativas. Así, proporcionados los diferentes instrumentos interpretativos, que además pueden variar con el tiempo, los jueces tienen discreción para elegir entre las diferentes opciones que integran el marco interpretativo correspondiente ${ }^{15}$.

Considero que la descripción que el realismo moderado hace de la práctica supone una distorsión de ella, que exagera el carácter controvertido de algunos casos que vienen remitidos y son resueltos en atención a los diferentes instrumentos interpretativos. Así, a partir de la constatación de que en la interpretación jurídica rigen diversos instrumentos interpretativos, no cabe concluir que los jueces siempre cuentan con múltiples opciones interpretativas. En este sentido, un defensor de la vigilia podría reconocer que hay múltiples instrumentos jurídicos, que estos dependen de la práctica del conjunto de intérpretes, y que pueden variar con el tiempo y, sin embargo, seguir sosteniendo que, respecto de cada juez individual y desde la perspectiva sincrónica, hay enunciados interpretativos verdaderos y el juez puede equivocarse. Enfatizar que, ya que se dan ciertos problemas interpretativos, las disposiciones expresan una multiplicidad de normas posibles nos hace perder de vista que hay numerosos supuestos que no suscitan dudas. Y no suscitan dudas precisamente porque hay una caracterización de la norma expresada por la disposición que no es problemática, sin ella dejaríamos de reconocer que el derecho es un instrumento capaz de guiar la conducta, al menos en alguna medida ${ }^{16}$.

Es importante advertir además que la existencia de diferentes instrumentos interpretativos es contingente, así como también lo es que el sistema jurídico confiera al juez discreción para escoger entre los diferentes instrumentos. Entonces, la tesis realista no puede

medio de transporte o solo, por ejemplo, medios de transporte motorizados. Y la problemática relativa a la disposición constitucional "El presidente firma los decretos" puede ser reconstruida como un supuesto en el que tenemos dudas acerca de si el caso en que el Presidente no quiere firmarlo constituye o no una infracción del precepto. Además, en ambos casos, en el planteamiento de las diferentes posibilidades y en su resolución, el juez cuenta con diversos instrumentos interpretativos, por lo que no parece que en la tarea del juez la interpretación orientada a hechos sea una actividad esencialmente distinta de la interpretación en abstracto. Sosteniendo este argumento, véase Ramírez Ludeña 2012: 102 y ss.

${ }^{15}$ Guastini (2008: 143-149) señala que hay diferentes elementos que hacen verdadera la tesis escéptica: la equivocidad, derivada de los instrumentos interpretativos, pero también las teorías dogmáticas y los sentimientos de justicia de los intérpretes. Por lo demás, según Guastini (2008: 143 y ss.) las disposiciones expresan varias normas, pero ello no abarca solo los casos en que hay dudas acerca de si una disposición expresa N1 o N2, sino otros casos como la derrotabilidad, o la aplicación del argumento a contrario.

${ }^{16}$ Ramírez Ludeña 2012: 95 y ss. 
ser conceptual, sino sociológica, acerca de lo que ocurre en nuestros sistemas jurídicos, por lo que la mera existencia de una pluralidad de instrumentos interpretativos no permite fundamentar que los enunciados interpretativos nunca tienen valor de verdad. Además (como se señaló con anterioridad), en sistemas como los nuestros, en que sí existen múltiples instrumentos interpretativos, numerosos supuestos son resueltos del mismo modo en atención de los diferentes instrumentos interpretativos. Y, en aquellos casos en que los diferentes instrumentos sí conducen a diferentes soluciones, puede consolidarse una determinada interpretación. Entonces, no podemos pasar de afirmar que en los sistemas contemporáneos existen numerosos instrumentos interpretativos, a sostener que las disposiciones siempre expresan diversas normas.

\subsection{La existencia de un marco de interpretaciones}

Pese a lo anteriormente señalado, la discrepancia acerca del número de casos controvertidos por la incidencia de los instrumentos interpretativos no puede constituir el elemento central de controversia entre las dos concepciones por lo que respecta a la verdad de los enunciados interpretativos. Ello es así debido a que la diferencia en el número de supuestos problemáticos no permite fundamentar por sí sola que no hay enunciados interpretativos verdaderos o falsos, al menos en determinados casos. En este sentido, podría sostenerse que, aunque haya un marco de posibilidades interpretativas, hay enunciados interpretativos falsos en aquellos casos en que se llevan a cabo interpretaciones fuera del marco. También cabría defender que hay enunciados interpretativos verdaderos si se trata de enunciados comprendidos en el marco de interpretaciones admisibles. Asimismo, si tenemos en cuenta que los partidarios del realismo moderado admiten la posibilidad de que se consoliden determinadas interpretaciones y que hay interpretaciones pacíficas, se requieren argumentos adicionales para fundamentar que, en tales casos, no hay enunciados interpretativos verdaderos. ¿Por qué no entender que, cuando se consolidan determinadas interpretaciones, o si las interpretaciones son pacíficas, los enunciados interpretativos son verdaderos? Rechazar la posibilidad de atribuir valor de verdad en cada uno de los casos anteriores requiere de argumentos adicionales.

Empezaré con la primera de las posibilidades (los enunciados interpretativos que hacen referencia a interpretaciones fuera del marco son falsos) y la conectaré con las otras dos. Aumque Guastini considera que hay un marco de interpretaciones posibles, se diferencia del realismo radical al entender que no todo puede tener cabida ${ }^{17}$. Sin embargo, ¿por qué

${ }^{17}$ Así, ya hemos visto que Guastini diferencia entre interpretación decisoria y creativa, en función de si la opción interpretativa está comprendida o no en el marco de interpretaciones admisibles. La decisoria es análoga a la redefinición, y la creativa a la estipulación. En este sentido, véase, por ejemplo, Guastini 2010: 78 y 79. Su asunción de la existencia de una diferencia entre creación e interpretación, así como su aceptación de que las interpretaciones posibles pueden ser descritas, permite descartar una de las posibles estrategias para considerar que los enunciados interpretativos carecen de valor de verdad: que los significados no existen. E incluso aunque esa fuera la estrategia, del mismo modo que puede entenderse -siguiendo la reconstrucción de Russell- que el enunciado "El rey de Francia es calvo" es falso, y no carente de valor de verdad, podría considerarse que si los significados no existieran, los enunciados interpretativos serían sistemáticamente falsos, y no carentes de valor de verdad. En este sentido, véase Arena 2013. 
no considerar entonces (como así lo hace la vigilia) que los enunciados interpretativos que recogen interpretaciones fuera del marco son falsos? Esta parece ser una reconstrucción en principio plausible, en el sentido de que nuestra primera reacción ante un enunciado de ese tipo es afirmar que es falso ${ }^{18}$. La concepción realista moderada podría ofrecer (al menos) dos respuestas: 1) Del mismo modo en que no puede afirmarse que un enunciado interpretativo es verdadero o falso cuando el juez selecciona una de las interpretaciones posibles, ya que está escogiendo una posibilidad entre otras, cuando el juez opta por una posibilidad que está fuera del marco, también está haciendo una selección, una adscripción, por lo que no cabría hablar de verdad o falsedad; 2) Del mismo modo que ocurre cuando se ha consolidado una determinada interpretación, el realismo moderado podría sostener que en tales casos sí puede hablarse de verdad o falsedad, pero porque no estamos ante un genuino enunciado interpretativo, sino ante un enunciado que describe interpretaciones. Analizaré a continuación cada una de esas posibilidades.

Empecemos con la primera de las respuestas. Guastini señala que los enunciados interpretativos carecen de valor de verdad porque en el sistema existen varias posibilidades interpretativas y el juez opta por una de ellas. Habría que plantearse entonces si, cuando el juez profiere (o asume) un enunciado interpretativo con la forma " $X$ significa $Y$ ", el enunciado carece de valor de verdad debido a que existen otras posibilidades, en lugar de considerar que es verdadero o falso.

Imaginemos que en un determinado sistema jurídico existen diferentes posibilidades interpretativas respecto de un término o disposición $(X)$. Así, en la comunidad en cuestión se emplean los instrumentos 1 y 2 , que conducen a las interpretaciones $Y$ y $Z$, respectivamente. Entonces, cuando el juez afirma una de esas interpretaciones (cuando profiere o asume " $X$ significa $\left.Y^{\prime \prime}\right)$ habría al menos dos modos de entender lo que ocurre.

Supongamos que entendemos que el sistema jurídico contiene una conjunción de interpretaciones. En tal caso, el enunciado interpretativo " $X$ significa $Y$ " sería verdadero, y no un enunciado carente de valor de verdad. Del mismo modo, sería verdadero un enunciado interpretativo que señalara que $X$ significa $Z$. Y el enunciado " $X$ significa $W$ " sería falso ${ }^{19}$.

$\mathrm{Si}$, en cambio, entendemos que en el sistema jurídico existe una disyunción entre diferentes posibilidades interpretativas ${ }^{20}$, ello tampoco nos lleva, por sí solo, a sostener que los enunciados interpretativos carecen de valor de verdad. Pensemos en un sistema jurídico que, respecto de un término o disposición $(X)$, admite dos significados posibles, $Y$ o $Z$. Los

${ }^{18}$ Esta parecería ser la reconstrucción más plausible porque, según el realismo moderado, se trata de una creación y no propiamente de una interpretación. En este sentido, si un juez afirma " $X$ significa $Y$ ” y en lugar de interpretar está en realidad creando un nuevo significado, resulta intuitivo sostener que el enunciado es falso, y no carente de valor de verdad.

${ }^{19} \mathrm{Y}$ si se entendiera que el enunciado interpretativo señala que $X$ solamente significa $Y$, el enunciado sería falso, y no carente de valor de verdad. En un sentido similar, Arena (2013) ha sostenido que no puede decirse que los enunciados interpretativos carecen de valor de verdad porque asocian a una disposición un único significado, cuando en realidad las disposiciones poseen varios significados. En tal caso los enunciados interpretativos serían falsos, no carentes de valor de verdad.

${ }^{20}$ De hecho, Guastini asume que cuando existen múltiples instrumentos interpretativos estamos ante una disyunción de interpretaciones en Guastini 1999: 102. 
enunciados " $X$ significa $Y$ ” y " $X$ significa $Z$ " serían verdaderos o falsos, y un enunciado que señalara que $X$ significa $W$ sería falso. En otras palabras, si tenemos una disyunción entre dos interpretaciones posibles $(Y, Z)$, aunque sabemos que es verdad que $X$ significa $Y$ o $Z$, no sabemos si es verdad que significa $Y$ ni sabemos si es verdad que significa $Z$, pero sabemos que una de las dos cosas es verdadera (" $X$ significa $Y$ ” y " $X$ significa $Z$ ” no pueden ser ambos falsos); además, sostener que $X$ significa $W$ sería falso. En todo caso, si existe una disyunción de interpretaciones, $\mathrm{u}$ concreto $\mathrm{n}$ enunciado interpretativo es verdadero o falso, y no carente de valor de verdad ${ }^{21}$.

\subsection{La dimensión adscriptiva de la interpretación}

Quizá el argumento central del realismo moderado para rechazar que los enunciados interpretativos tengan valor de verdad tiene que ver con la dimensión adscriptiva de la interpretación. De hecho, Guastini rechaza que los enunciados interpretativos sean enunciados prescriptivos. Así, objeta que la cuestión se plantee a modo de disyuntiva entre descripción y prescripción, y enfatiza la relevancia de atender a la naturaleza adscriptiva de los enunciados interpretativos. No obstante, este carácter adscriptivo puede ser entendido de diversos modos y, como trataré de explicar, ninguno de ellos es suficiente para mostrar que los enunciados interpretativos carecen de valor de verdad ${ }^{22}$.

\subsubsection{La arbitrariedad del lenguaje}

Es comúnmente aceptado que las palabras no están vinculadas de manera natural con sus significados ${ }^{23}$. De este modo, sería extraño cuestionar que la conexión entre ambos elementos es arbitraria y que depende de que exista una práctica de atribución de significado -ya sea por relacionar la palabra con un sentido o por emplearla para hacer referencia

21 Aunque existen diferencias notables en función de si se entiende que el sistema contiene una disyunción incluyente o excluyente, en cualquiera de los dos casos el enunciado interpretativo es verdadero o falso, no carente de valor de verdad. Es importante advertir que, ya se sostenga que el derecho contiene una conjunción o una disyunción de interpretaciones, la vigilia también se vería afectada por las consideraciones anteriores: salvo que se aporten argumentos adicionales, en los casos difíciles los enunciados interpretativos son verdaderos o falsos, y no carentes de valor de verdad. No obstante, podría ocurrir que haya casos difíciles en que el significado de un texto resulte no simplemente problemático, porque hay más de un significado admisible, sino que se trate de una cuestión enteramente indeterminada. Cabría entonces entender que, en ese tipo de casos difíciles, los enunciados interpretativos carecen de valores de verdad. En un sentido similar, Arena (2013: 7, n. 12) ha señalado que el realista moderado podría sostener que es indeterminado cuáles son los significados que posee un texto normativo. Pero ello conllevaría que ni siquiera el resultado de la interpretación cognitiva poseyera valor de verdad, debido a que ningún elenco de significados puede describir un conjunto indeterminado. Respondiendo a esa cuestión, Guastini (2012: 184, n. 15) ha sostenido que, aunque identificar todos los significados posibles de un texto normativo sería probablemente imposible, desde el punto de vista sincrónico los significados posibles de un texto son, si bien numerosos, finitos.

22 Guastini 2010: 170.

${ }^{23}$ Incluso quienes entienden que puede afirmarse que descubrimos los significados niegan que las palabras estén ligadas a esos significados con independencia de que haya una práctica en que se usan determinadas palabras para referir a ciertos objetos. En este sentido, véase Kripke i980 y Putnam 1975. 
a determinados objetos. Por ejemplo, el término "perro" tiene un determinado significado fruto de la atribución de significado en la comunidad relevante. Sin embargo, lo anterior, por sí solo, no nos conduce a negar que las afirmaciones respecto de los significados tengan valor de verdad. Las afirmaciones relativas al significado de los términos son verdaderas o falsas en virtud del uso general que se haga del término y no de cada individuo en particular. De modo similar, puede defenderse que las prácticas interpretativas en el ámbito jurídico -que, como señala Guastini, presentan ciertas peculiaridades respecto de las prácticas lingüísticas cotidianas- determinan (al menos en ocasiones, si no hay problemas de indeterminación) el valor de verdad de los enunciados interpretativos. Así, aunque un juez individual pueda proferir un enunciado interpretativo falso si no se ajusta a la interpretación de la comunidad, la comunidad interpretativa en su conjunto no puede hacerlo, en el sentido de que es ella la que determina qué enunciados son verdaderos, y cuáles son falsos. Entonces, si bien con carácter individual los diferentes intérpretes no determinan las interpretaciones de los textos, la práctica en su conjunto sí las determina, por lo que los enunciados interpretativos de los jueces individualmente considerados pueden ser verdaderos o falsos ${ }^{24}$.

\subsubsection{El juez puede escoger como cuestión de hecho}

El argumento central para negar que los enunciados interpretativos son verdaderos o falsos podría ser no que el significado depende de un conjunto de adscripciones, sino que, en el caso del juez particular, se produce siempre una adscripción de significado. En este sentido, los realistas podrían sostener que, incluso aunque el juez adopte una interpretación no problemática, en todo caso decide conformarse al estándar comunitario. Habría entonces dos sentidos de "adscripción" diferentes. Por un lado está la adscripción generalizada de significados a los términos, de la que depende que las palabras expresen determinados significados y refieran a determinados objetos. Por otro lado están las concretas adscripciones del juez al llevar a cabo sus interpretaciones. No obstante, tampoco este último parece ser un argumento concluyente. El hecho de que siempre exista la posibilidad por parte del juez individual, e incluso por parte de toda la comunidad, de haber actuado de otro modo, no impide que los enunciados tengan valor de verdad. Así, el hecho de que cuando digo "mi perro está en el sofá", podría haber empleado otras palabras, o las mismas palabras con otro significado, no impide que digamos que, en virtud de la historia del uso del término y debido a nuestro conocimiento del mundo, mi enunciado sea verdadero o falso.

\subsubsection{El juez puede escoger como cuestión de derecho}

Un tercer sentido en que la interpretación del juez supone una adscripción se vincula con el hecho de que el juez tiene la facultad de elegir una interpretación entre otras posibles. Aquí la posibilidad no es meramente fáctica, sino normativa. Esto es, los enunciados

${ }^{24}$ De hecho, como hemos visto con anterioridad, Guastini reconoce que sí puede predicarse la verdad en el caso de los enunciados subsuntivos. Si ello es así, y teniendo en cuenta que en tales supuestos también se producen adscripciones de significados, la adscripción por sí sola no parece determinante. 
interpretativos carecerían de valor de verdad a causa de que no se estaría describiendo una opción interpretativa entre otras, sino que se estaría optando por una de las interpretaciones posibles en términos jurídicos. El problema de considerar que este es el sentido relevante de adscripción, que determina que los enunciados interpretativos carecen de valor de verdad, es que no explica el desacuerdo entre el formalismo moderado y el realismo moderado. Esto es así ya que también el formalismo moderado sostiene que, cuando existen diversas opciones interpretativas y el juez tiene discreción para escoger una de ellas, los enunciados interpretativos carecen de valor de verdad.

\subsection{El juez usa la interpretación}

En un ulterior sentido de "adscripción”, podría sostenerse que los enunciados interpretativos carecen de valor de verdad, incluso si no hay dudas interpretativas, aunque los jueces no pueden contentarse con describir las interpretaciones, sino que tienen que usarlas para calificar el supuesto de hecho que les es presentado ${ }^{25}$. Sin embargo, si bien es cierto que los jueces aplican la norma jurídica resultante de la interpretación al caso en cuestión, ello no obsta a que el propio enunciado interpretativo sea descriptivo y tenga valor de verdad. Es decir, si bien en el razonamiento del juez este usa la interpretación para acabar "adscribiendo" (en un sentido laxo) una consecuencia jurídica a un caso espacio-temporalmente determinado, ello no impide que el propio enunciado interpretativo que es parte de ese razonamiento sea verdadero o falso. Del mismo modo, aunque el juez haga uso de la premisa fáctica en su razonamiento, ello no impide que el propio enunciado acerca de los hechos sea verdadero o falso. Como veremos más adelante en este trabajo, resulta cuestionable que un enunciado interpretativo sea meramente una descripción -y, por ello, es cuestionable que el enunciado interpretativo sea equivalente a la premisa fáctica-; pero me interesa en este punto enfatizar que el mero hecho de formar parte del razonamiento jurídico no hace que carezca de valor de verdad ${ }^{26}$.

${ }^{25}$ Guastini 1999: 204.

${ }^{26}$ Podría insistirse en que el realismo moderado entiende que la diferencia es pragmática: el juez tiene otras opciones y decide conformarse a una de ellas al proferir el enunciado interpretativo, introduciendo un cambio en el mundo. Sin embargo, este modo de ver las cosas no me parece sustancialmente diferente de los señalados con anterioridad, y ya criticados. Así, si bien es obvio que el juez puede (salvo que se asuma alguna forma de determinismo) hacer otra cosa, ello es irrelevante para la discusión. También puede hacer otra cosa respecto de la premisa fáctica, y ello no impide que esta sea verdadera o falsa. Además, tampoco se entiende qué incidencia tiene que se produzcan ciertos cambios en el mundo o que se generen ciertas consecuencias jurídicas (como también ocurre cuando el juez se pronuncia respecto de la premisa fáctica, y no rechazamos por ello que tenga valor de verdad). Finalmente, y como ya he señalado anteriormente, que los enunciados interpretativos carezcan de valor de verdad si existen diversas opciones jurídicas es algo que no rechazaría la vigilia. Por lo demás, podría defenderse que los enunciados interpretativos son en realidad reglas constitutivas, carentes de valor de verdad. Aunque no me parece implausible entender que los instrumentos interpretativos son reglas constitutivas, parece extraño entender que lo son los propios enunciados interpretativos, especialmente en los casos fáciles. Así, parece absurdo que, frente a una mesa, yo profiera "esto cuenta como una mesa". Además, y dejando de lado las dificultades para determinar cuándo nos hallamos ante una regla constitutiva (¿depende meramente de las intenciones de quien la profiere, de otras reglas, del contexto?), entender que los enunciados interpretativos son reglas constitutivas no permitiría al realista moderado distinguir la interpretación de la creación. 
1.5. Interpretaciones no problemáticas y que se consolidan

Pasemos ahora a la segunda de las respuestas que planteé con anterioridad en este trabajo: el realismo moderado podría rechazar que los enunciados que recogen interpretaciones fuera del marco tienen valor de verdad, del mismo modo que no admite que cuando se consolidan determinadas interpretaciones pueda afirmarse que hay enunciados interpretativos con valor de verdad. En tales casos, Guastini sostiene que puede hablarse de verdad o falsedad de los enunciados, pero que no estamos ante genuinos enunciados interpretativos, sino ante enunciados que describen interpretaciones. Entonces, la existencia de estos casos no contaría a favor de la vigilia porque no serían supuestos en que los enunciados interpretativos son verdaderos o falsos, sino enunciados descriptivos de interpretaciones.

Guastini sostiene que, aunque pueden variar con el tiempo, en ocasiones hay interpretaciones que se consolidan y además las controversias interpretativas no suelen afectar al significado de un texto normativo en su totalidad ${ }^{27}$. Pero ¿no habría entonces que reconocer que hay enunciados interpretativos verdaderos desde la perspectiva sincrónica? Como he señalado, el realismo moderado asume que no, porque se trataría de enunciados descriptivos de interpretaciones, no genuinos enunciados interpretativos ${ }^{28}$. No obstante, esto es bastante extraño. Así, no se entiende en qué se diferenciarían estos casos de los enunciados subsuntivos en los casos claros, que Guastini sí admite que pueden tener valores de verdad, en tanto enunciados genuinamente subsuntivos y no como enunciados que describen subsunciones.

Estos casos tienen una importancia crucial en la discusión que nos ocupa, ya que la diferencia central entre las distintas concepciones (realismo moderado y formalismo moderado) parece radicar en el análisis de los casos en que se han consolidado determinadas interpretaciones y en los casos de interpretaciones pacíficas. Ambas posiciones entienden que hay enunciados interpretativos que carecen de valor de verdad en los casos en que hay diferentes opciones interpretativas. Si Guastini entiende además que hay interpretaciones consolidadas e interpretaciones pacíficas, ¿por qué no admitir que en esos casos los enunciados interpretativos son verdaderos, y no que se trata de enunciados descriptivos de las interpretaciones consolidadas y pacíficas? Pero la cuestión no es solo que es problemático justificar la diferenciación anterior, sino que además Guastini "haría verdadera” la tesis del realismo moderado simplemente mediante una redefinición de lo que es un enunciado interpretativo, que supondría la elección de una interpretación entre otras posibles. Habría

${ }^{27}$ Guastini 2010: 134-137. De este modo parecería que Guastini admite que, al menos en algunos casos, la interpretación judicial es un acto de descubrimiento y no de voluntad.

${ }^{28}$ Según Guastini (2010: 132 y ss.), cuando se consolida una interpretación (y en todo caso respecto de partes de las disposiciones que no son problemáticas en términos interpretativos), a las proposiciones jurídicas subyacen no enunciados interpretativos verdaderos sino enunciados verdaderos acerca de interpretaciones. Así, para Guastini $(2012,194$, n. 38) si existe una interpretación consolidada de un enunciado normativo, es verdadero el enunciado que la describe (el enunciado que describe la interpretación vigente) y no el enunciado interpretativo mismo. En todo caso, reconocer que en tales casos existen interpretaciones que se consolidan, y que pueden ser descritas, supone, de nuevo, que Guastini no se compromete con que los significados no existen. Entonces, ese no puede ser el fundamento último para rechazar que los enunciados interpretativos tienen valor de verdad. 
solo un desacuerdo aparente entre la posición de Guastini y la vigilia, debido a que el formalista moderado también admitiría que, en los casos de elección entre diversas opciones interpretativas, los enunciados interpretativos carecen de valor de verdad.

\subsection{Las interpretaciones cambian}

Tampoco es un argumento concluyente contra el valor de verdad de los enunciados interpretativos el sostener que las interpretaciones cambian. Es decir, el hecho de que las interpretaciones que se consolidan puedan variar, y que también pueda hacerlo el marco de interpretaciones admisibles, todavía no nos permite concluir que no hay enunciados interpretativos verdaderos. A efectos de caracterizar adecuadamente nuestras intuiciones compartidas acerca del lenguaje en general y de la interpretación jurídica en particular, es importante tomar en consideración dos perspectivas: la sincrónica y la diacrónica. Resultaría extraño discutir que la comunidad interpretativa puede hacer que lo que antes (en el momento t1) constituía una interpretación posible o consolidada del término, ahora (en el momento t2) no lo sea. En este sentido, desde la perspectiva diacrónica las interpretaciones pueden variar, lo que no impide que hagamos referencia a las interpretaciones en cada uno de los momentos (esto es, si atendemos a la perspectiva sincrónica). Así, aunque haya cambios en las interpretaciones, esto no varía la forma de analizar las interpretaciones previas, que dependían de las consideraciones de la comunidad en su momento. Entonces, puede afirmarse que si bien desde el punto de vista sincrónico hay enunciados interpretativos verdaderos y los intérpretes individuales pueden equivocarse, desde la perspectiva diacrónica los instrumentos interpretativos pueden variar, si se producen los cambios correspondientes en la comunidad interpretativa, cambiando también qué enunciados interpretativos son verdaderos ${ }^{29}$. En lo que aquí interesa, los enunciados interpretativos podrían seguir siendo verdaderos o falsos si atendemos a la perspectiva sincrónica, lo que no se vería afectado por lo que ocurre a nivel diacrónico. En todo caso, no estamos ante un problema distinto del que ya he venido analizando a lo largo del trabajo.

\subsection{Siempre hay consecuencias jurídicas...}

Quizá el elemento determinante sea que, de acuerdo con los realistas, el juez puede optar por cualquiera de las interpretaciones, porque la que escoja, esté o no dentro del marco, se aleje o no de la interpretación consolidada, tendrá consecuencias jurídicas. Sin embargo, no es esta la posición de Guastini ni del realismo moderado: precisamente porque pretenden distanciarse de las versiones radicales del realismo, no defienden que el reconocimiento de

\footnotetext{
${ }^{29}$ Guastini (2008: 17 y 18) encuentra problemática la teoría hartiana porque los casos no son objetivamente claros o difíciles. Pero a ello puede responderse que, si bien la caracterización depende de la comunidad en su conjunto y que puede variar diacrónicamente, sincrónicamente los casos son claros o difíciles. Si Guastini no aceptara que, pese a que las interpretaciones cambian, ello no altera qué interpretaciones son admisibles y cuáles se han consolidado en un momento dado, entonces difícilmente su posición podría ser diferenciada del realismo extremo.
} 
efectos a las interpretaciones fuera del marco suponga que se trata de genuinas interpretaciones. Para Guastini, pese a que se produzcan efectos, se tratará de un caso de creación, y no de interpretación ${ }^{30}$. Por tanto, que algo sea un caso de creación o de interpretación no depende de que se declare o no su invalidez o de que sea ineficaz. Tampoco parece ser entonces determinante, a efectos de considerar que los enunciados interpretativos tienen valor de verdad, que se produzcan consecuencias jurídicas (como tampoco lo es respecto del valor de verdad de la premisa fáctica).

\subsection{Las actitudes de los intérpretes}

Otra posibilidad es que los enunciados interpretativos carezcan de valor de verdad por las actitudes de los jueces respecto de la interpretación. Supongamos que los jueces no solo se comportan de un determinado modo al identificar el derecho, sino que además expresan ciertas actitudes. Y supongamos que ello también se produce respecto de las interpretaciones $^{31}$. Entonces, podría sostenerse que los enunciados interpretativos carecen de valor de verdad porque los jueces expresan, al proferirlos, determinadas actitudes. Sin embargo, creo que ello tampoco permitiría fundamentar el rechazo del valor de verdad de los enunciados interpretativos. Así, podría entenderse que los jueces tienen, con carácter general, ciertas actitudes respecto de las interpretaciones y, sin embargo, cabría sostener además que el juez individual puede describir, mediante un enunciado interpretativo, el estándar resultante de esa actitud convergente.

${ }^{30}$ Por ello, la aceptación posterior por parte del conjunto de intérpretes no supondrá, desde el punto de vista de Guastini, que esa sea una interpretación posible. Si se consolida, esa interpretación se incorporará al marco de posibilidades, lo que no cambia que en un primer momento no sea una genuina interpretación. Aunque Guastini no lo señala, parece que apartarse del marco de soluciones posibles, de la única interpretación consolidada o de las interpretaciones pacíficas constituyen casos de error en tanto el juez no lleva a cabo una genuina interpretación y en este sentido puede decirse que actúa incorrectamente. Analizaré la cuestión de la corrección más adelante en este trabajo.

31 Además, y aunque ello no forma parte del argumento principal de este trabajo, considero que las condiciones de existencia de un sistema jurídico no pueden agotarse en la práctica de identificar textos sin una actitud crítico-reflexiva respecto de ciertos modos admisibles de otorgarles un contenido. Imaginemos que un individuo considera que el Código Civil español es parte del derecho español, pero entiende que su significado debe serle atribuido a partir de un programa informático que asigna significados al azar. Imaginemos que otro individuo considera que su contenido depende de lo que su hijo de diez años dice que expresa. Finalmente, un tercer individuo entiende que aquello que expresa el Código Civil depende del lenguaje ordinario. ¿Diríamos que hay un acuerdo entre los diferentes individuos acerca de que el Código Civil es parte del derecho si no hay un acuerdo básico acerca de lo que expresa el Código? Si cada uno, siguiendo su propio criterio, sostiene una interpretación radicalmente diferente de la de los demás respecto de lo que establece el Código Civil, parece que invocar el Código Civil se torna algo superfluo. Dicho en otros términos, si la actividad interpretativa no es constitutiva de la actividad jurídica, la convergencia en las fuentes podría producir los mismos resultados que su ausencia (un total desacuerdo acerca de qué normas son válidas) con lo que podría ser un hecho completamente irrelevante. Esta idea es sugerida por Diego Papayannis (2014). En el análisis anterior he dejado de lado la difícil cuestión de si los instrumentos interpretativos son parte de la regla de reconocimiento o si van necesariamente unidos a ella. 
Otro modo de plantear la cuestión consiste en destacar que el juez no solo describe el estándar resultante de la conducta y actitud convergente del resto de jueces, sino que al proferir o asumir un determinado enunciado interpretativo en su razonamiento también tiene cierta actitud, en tanto participante de la práctica jurídica. Entonces, podría sostenerse que el enunciado interpretativo carece de valor de verdad, al expresar una determinada actitud por parte del juez y no tener un carácter descriptivo.

Puede ser interesante trazar aquí un paralelismo respecto de los enunciados normativos con carácter general. Así, se ha discutido si enunciados como "Está permitida la entrada de perros a la universidad" constituyen una descripción o una manifestación de ciertas actitudes. En este ámbito, suele distinguirse entre enunciados internos y externos ${ }^{32}$. Mediante un enunciado interno el sujeto manifiesta su aceptación del estándar en cuestión. Los externos, en cambio, son proferidos por quien adopta una actitud teórica respecto del derecho, y describe, ya sea meras regularidades de conducta (enunciados externos extremos) o la aceptación por parte de los participantes de la práctica (externos moderados). ¿Qué ocurre en el caso de los jueces? Mediante un enunciado jurídico el juez profiere un enunciado interno, adoptando una actitud práctica en relación con el estándar que acepta ${ }^{33}$. Entonces, precisamente por ello, el enunciado carecería de valor de verdad. El juez que acepta tiene una actitud críticoreflexiva acerca de un estándar de comportamiento, lo que supone que está comprometido a actuar según la regla y a evaluar la conducta basada en ella. Dicha aceptación se manifiesta al proferir enunciados jurídicos comprometidos ${ }^{34}$.

32 Hart 1994: 102 y ss.

33 En realidad lo que ocurre es que el juez: a) expresa su aceptación de la regla de reconocimiento como el test para determinar qué normas forman parte del sistema; y b) expresa el juicio de que cierta regla pasa el test. Además, asume que la regla de reconocimiento no solo es aceptada por él, sino que es la regla de hecho aceptada y empleada en general en el sistema. Dejaré de lado ciertos aspectos problemáticos, como es si, para que exista un sistema jurídico, la aceptación debe darse también referente a las diferentes normas, o solo concerniente a las reglas secundarias, o si es necesario que los ciudadanos, y no solo los officials, acepten. Asimismo, no abordaré la cuestión de si la aceptación constituye una actitud general relativo al sistema en su conjunto, o atinente a sus normas (ya sean las primarias y secundarias o solo las secundarias). Tampoco me detendré en la cuestión de si la aceptación supone una determinada actitud moral, la creencia en que hay razones morales para aceptar, si exige considerar el estándar como jurídicamente correcto (como algo distinto de moralmente correcto) o si se puede aceptar por cualquier razón. Véanse, en este sentido, la posición de Hart acerca de la cuestión (Hart 1982: 153 y ss., y 257), y las apreciaciones de Holton (1998). Véase también el interesante análisis de Redondo (1999: capítulo 5), quien distingue entre aceptación, simulación, asunción, creencia e internalización.

${ }^{34}$ Como señala Hart (1994: 57), "What is necessary is that there should be a critical reflective attitude to certain patterns of behaviour as a common standard, and that this should display itself in criticism (including self-criticism), demands for conformity, and in acknowledgements that such criticism and demands are justified, all of which find their characteristic expression in the normative terminology of 'ought', 'must', and 'should', 'right' and 'wrong'. Que el enunciado sea o no comprometido no depende meramente de las intenciones del juez ni de si está o no comprometido realmente, sino de si la aceptación se expresa o está implícita en la proferencia del enunciado. Entonces, un aceptante de la regla de reconocimiento podría no expresar la aceptación al proferir un enunciado normativo (por estar meramente describiendo lo que el sistema establece). Más problemático me parece en cambio que un no aceptante con carácter general pueda manifestar su aceptación al proferir un determinado enunciado, simulando aceptar. 
Traslademos lo anteriormente señalado al caso de los enunciados interpretativos. Tendremos, por un lado, enunciados externos que describen interpretaciones. Por otro lado, enunciados interpretativos internos formulados o asumidos por un juez que acepta el estándar interpretativo ${ }^{35}$. Entonces, a la cuestión acerca de si los enunciados interpretativos tienen valor de verdad hay que responder que depende. Depende de las actitudes de quién profiere o asume el enunciado. Si nos centramos en el caso de los jueces, el enunciado puede ser proferido o asumido por un juez que acepta y que así lo expresa, y entonces cabría concluir que carece de valor de verdad. Pero también puede ser proferido o asumido por un juez que, aunque acepta, no lo expresa (sino que describe), en cuyo caso el enunciado sí tendría valor de verdad.

¿Puede entenderse que Guastini rechaza que los enunciados interpretativos tengan valor de verdad porque se basa en las actitudes de los jueces? No parece ser el caso, porque los realistas no parecen sostener que los jueces adoptan los instrumentos interpretativos como estándar de corrección. Pero entonces, siguiendo lo anteriormente señalado, los enunciados interpretativos serían descriptivos, y podrían ser verdaderos o falsos ${ }^{36}$. Así, el realista moderado parece enfrentarse a un dilema: o bien emplea el argumento de las actitudes para fundamentar el rechazo del valor de verdad de los enunciados interpretativos, pero entonces tiene que reconocer que tiene sentido hablar de corrección, o bien rechaza ese argumento (como parece ser el caso), pero entonces no cuenta con ese instrumento para rechazar que los enunciados interpretativos son verdaderos o falsos y, lo que es más importante, no puede diferenciar su posición de la del realismo radical. El realista podría seguir insistiendo en que, aunque no se estén incumpliendo reglas, lo relevante es que el juez que se aparta del marco interpretativo o de la interpretación consolidada no interpreta, sino que su actividad es creativa. Pero nada se seguiría de la distinción terminológica. Quien creara derecho no estaría actuando incorrectamente, sino que, simplemente, no estaría interpretando. Sin embargo, creo que dicha reconstrucción no le permitiría distinguirse del realismo radical, más que por una cuestión de cómo etiquetar los casos.

Otra posibilidad es que el realista moderado entienda que los jueces son participantes pero no aceptantes. Habría entonces jueces que son bad men, en el sentido de que no tienen una actitud crítico-reflexiva y actúan basados en consideraciones estratégicas. Pero si se admite que se puede aceptar sobre la base de consideraciones estratégicas, ¿no será el bad man un aceptante más? No, en tanto se diferencie el juez que acepta a base de consideraciones estratégicas, y considera el derecho como un estándar general de conducta (juez aceptante),

${ }^{35}$ Del mismo modo que señalé respecto de los enunciados jurídicos con carácter general, lo que ocurre en el caso de los enunciados interpretativos comprometidos es que el juez: a) expresa su aceptación de ciertos instrumentos interpretativos como el test para determinar qué interpretaciones forman parte del sistema; y b) expresa el juicio de que cierta interpretación pasa el test (es decir, que $X$ significa $Y$ ). Además, asume que esos instrumentos no son solo aceptados por él, sino que son los instrumentos de hecho aceptados y empleados en general en el sistema. Pese a lo que se acaba de señalar, podría decirse que en los casos claros, que constituyen el foco principal de atención en este trabajo, no se da propiamente una aceptación de los instrumentos interpretativos, sino de la interpretación en concreto. En todo caso, ello depende, de nuevo, de cómo se reconstruya lo que ocurre en los casos claros: ¿se comprende la regla? ¿Se interpreta a base de los instrumentos?

${ }^{36}$ Evidentemente esta conclusión resulta paradógica: los realistas sostienen que los enunciados interpretativos pueden ser verdaderos o falsos y los formalistas moderados que no lo son. 
del juez que, caso a caso, actúa sobre la base de consideraciones estratégicas (juez bad man, no aceptante). Pues bien, ¿qué ocurre cuando el bad man profiere un enunciado jurídico o un enunciado interpretativo? Se trata de enunciados internos, en el sentido de proferidos por un participante, pero externos en el sentido de que no expresan aceptación. En este ámbito, quizá resulte de utilidad recurrir a la noción de detached statement, mediante estos el juez no expresaría su compromiso con el estándar, sino que señalaría qué es lo que debe hacerse de acuerdo con el sistema. Se han ofrecido diversas caracterizaciones de estos enunciados, y no solo se trata de una cuestión controvertida en la literatura si los detached statements constituyen una categoría independiente de los enunciados internos y externos, sino que además no es sencillo determinar en qué consisten. Pero asumamos que un detached statement expresa el punto de vista del legal man, es decir, de un hipotético sujeto que acepta lo que el derecho establece. No se trata de enunciados acerca de las creencias de otras personas, ya que puede que nadie tenga tal creencia, o puede que el individuo esté expresando una muy rara opinión respecto de una cuestión difícil. Tampoco son enunciados condicionales, del tipo "si aceptas este punto de vista, debes...", porque establecen lo que es el caso desde el punto de vista relevante, como si fuera válido, o relativo a la hipótesis de que lo es, pero sin respaldarlo efectivamente. En todo caso, aunque entendiéramos que el realismo moderado sostiene que los jueces son bad men y profieren detached statements, ello no les permitiría fundamentar que los enunciados interpretativos carecen de valor de verdad, ya que los detached statements son descriptivos, y por lo tanto susceptibles de ser verdaderos o falsos ${ }^{37}$.

\section{Ni verdad ni enunciados interpretativos. El papel de la corrección de las interpretaciones}

Hemos visto que suele diferenciarse entre las distintas concepciones acerca de la interpretación jurídica en función de la posición que adoptan respecto de los enunciados interpretativos. No obstante, esto resulta de entrada sorprendente, ya que los enunciados interpretativos están generalmente implícitos en las sentencias judiciales y no es frecuente la reflexión respecto de su naturaleza. Entre los teóricos del derecho, en general no se han desarrollado detallados argumentos acerca de qué es un enunciado interpretativo y de cuál sería la principal justificación para afirmar o negar que los enunciados interpretativos tengan valor de verdad. Por ello, considero que es relevante indagar acerca de las posibles razones para fundamentar o rechazar que los enunciados interpretativos tengan valor de verdad, pero también plantearse si tiene sentido que las diferentes concepciones interpretativas sean caracterizadas a base de ese elemento.

Partiendo de la concepción realista moderada de la mano de la posición de Riccardo Guastini, he analizado dónde podría radicar el punto de desacuerdo con el formalismo

${ }^{37}$ Acerca de los detached statements, véase Raz 1979: 153 y ss. y 1980: 234 y ss. y Hart 1982: 154 y ss. Una noción similar es la introducida por Shapiro (2011: 184 y ss.) cuando hace referencia a los enunciados desde una perspectiva. De acuerdo con Shapiro, se trata de descripciones de una perspectiva según la cual el derecho es moralmente legítimo. Aunque Shapiro sí se refiere al juez bad man, Raz introdujo la noción para hacer referencia a los enunciados proferidos por los abogados. Criticando la noción, véase, por ejemplo, Duarte D’Almeida 2011, Holton 1998, Muffato 2013, Toh 2007. 
moderado de corte hartiano por lo que respecta al valor de verdad de los enunciados interpretativos. Ninguna de las consideraciones expuestas por Guastini en sus distintos trabajos parece justificar el rechazo del valor de verdad de esos enunciados. Ello, evidentemente, no fundamenta que sí tengan valor de verdad, pero pone de manifiesto que la cuestión es altamente problemática. Y, lo que es más importante, la exposición de los diversos argumentos deja constancia de que puede controvertirse que se trace la distinción entre las concepciones en atención a ese elemento, que no es central a sus posiciones interpretativas y que, como hemos visto, no hace inteligible algunas de las discusiones. Así, si a nivel metateórico es preferible adoptar criterios para diferenciar entre teorías que recojan sus aspectos centrales y que hagan inteligibles los desacuerdos entre las distintas posiciones, el valor de verdad de los enunciados interpretativos parece un mal candidato.

Entre las principales conclusiones del camino recorrido, conviene destacar una particularmente sorprendente: no parece que exista un genuino desacuerdo entre las distintas concepciones. Esto es así porque, si tomamos como elemento de referencia el sentido que Guastini confiere a la expresión "enunciado interpretativo", el formalismo moderado no tiene por qué desacordar con su posición. Si entendemos que un enunciado interpretativo supone la elección de una interpretación cuando existen diferentes posibilidades interpretativas, la concepción hartiana estaría de acuerdo en que, en esos casos, los enunciados interpretativos carecen de valor de verdad. Precisamente, si existen diferentes opciones interpretativas el caso es difícil y el formalismo moderado acordaría con Guastini en que no puede atribuirse verdad o falsedad a esos enunciados interpretativos. En los casos en que sí parece haber un verdadero desacuerdo, esto es, en los casos que no son problemáticos a nivel interpretativo, Guastini entiende que lo que serían verdaderos o falsos serían los enunciados que describen interpretaciones. La concepción formalista moderada podría, sin embargo, no tener problemas en conceder ese punto, ya que, en una versión sofisticada, la vigilia podría reconocer que existen numerosos instrumentos interpretativos, que dependen de toda la comunidad y son cambiantes, pero que, desde el punto de vista del juez individual y desde la perspectiva sincrónica, hay enunciados interpretativos verdaderos que describen la única interpretación admisible en esa comunidad, en ese momento dado. No se ve qué se gana señalando que, en tales supuestos, no se trata de genuinos enunciados interpretativos, sino de enunciados que describen interpretaciones y, en todo caso, no habría entonces un genuino desacuerdo porque lo que para la vigilia sería un enunciado interpretativo, para el realismo sería un enunciado que describe interpretaciones.

En la última parte del trabajo he planteado si el elemento central que permitiría diferenciar las posiciones en relación con su posición respecto de los enunciados interpretativos es que el juez tiene determinadas actitudes al proferir o asumir el enunciado. No obstante, atender a este aspecto conduciría a una situación paradójica: el realismo moderado tendría que sostener que los jueces aceptan (y ello ser rechazado por el formalismo moderado). Además, el análisis de las actitudes de los jueces nos condujo a otra conclusión sorprendente: los enunciados interpretativos tienen o no valor de verdad en función del juez que los profiera o los presuponga en su razonamiento, y de lo que asume o expresa cuando los profiere o asume.

¿Por qué no diferenciar, en cambio, las posiciones en atención a si hacen referencia o no a la corrección de las interpretaciones de los jueces, en lugar de en atención al valor de verdad 
de los enunciados interpretativos ${ }^{38}$ ? Ello permitiría ordenar el debate entre los diferentes autores con independencia de si entienden que los enunciados interpretativos (acerca de los que raramente se pronuncian y que no son centrales en el debate ni para los propios participantes) son descriptivos, adscriptivos, etcétera ${ }^{39}$. Estarían quienes admiten el discurso acerca de la corrección, y quienes lo rechazan. Así, de un lado tendríamos a los realistas radicales (que creen que los jueces no pueden equivocarse), y del otro a los formalistas radicales y a los moderados (que se diferenciarían en virtud de si entienden que siempre, o solo a veces, pueden equivocarse).

¿Qué ocurriría con el realismo moderado? Es posible reconstruirlo de diferentes modos. Podría sostener que su posición no toma en cuenta el punto de vista interno de los participantes, si aceptan o no, sino que su análisis es externo. Ello supone no ofrecer un discurso en términos de corrección, pero también que el realismo moderado no pueda ser considerado una concepción interpretativa en pugna con las demás, al situarse en un nivel diferente de análisis.

Si se entendiera en cambio que el realismo moderado defiende que el juez puede equivocarse, aunque en los casos difíciles cuenta con distintas opciones, la diferencia entre esta posición y el formalismo moderado vendría dada únicamente por el número de supuestos problemáticos. De esta manera, podría considerarse que, si bien ambas posiciones reconocen que hay diferentes instrumentos interpretativos y que hay casos problemáticos, el realismo moderado insiste en que los jueces disponen generalmente de diversas opciones interpretativas, mientras que el formalismo moderado lo considera un fenómeno marginal ${ }^{40}$.

Lo fundamental entonces, a efectos de avanzar en el debate, será la indagación empírica acerca del número de casos problemáticos. Y, lo que resulta determinante a efectos de llevar a cabo la indagación empírica correspondiente, teorizar previamente acerca de cuáles son los sujetos relevantes a efectos interpretativos. Ello es así porque el realismo moderado parece asumir una concepción más amplia acerca de los sujetos relevantes, incluyendo a abogados y a dogmáticos, a efectos de considerar un caso como interpretativamente difícil. Pero al mismo tiempo de plantear la cuestión en términos de la verdad de los enunciados interpretativos, se ha centrado exclusivamente en lo que ocurre en sede judicial cuando el juez profiere o asume un enunciado interpretativo. Para una concepción formalista moderada de corte hartiano, en cambio, la relevancia de otros sujetos depende de que los funcionarios de justicia, que son los sujetos relevantes, les concedan esa incidencia. Al mismo tiempo,

\footnotetext{
${ }^{38}$ Otra opción sería centrar el debate en el nivel de las proposiciones interpretativas, esto es, en atención a si los autores entienden que las afirmaciones acerca de las interpretaciones son siempre verdaderas o falsas, o si, al menos en algunos casos, carecen de valor de verdad. No obstante, distinguir de este modo las posiciones también me parece problemático, ya que depende del posicionamiento en cuestiones como la bivalencia, la lógica difusa, etcétera. Así, por ejemplo, autores que tradicionalmente son agrupados como partidarios de la vigilia podrían ser agrupados por separado si uno rechaza y otro suscribe la bivalencia.

${ }^{39}$ Hablar de corrección posibilita además incorporar en el debate aquellas concepciones que, pese a sostener que siempre hay casos de interpretaciones correctas o incorrectas, entienden que no tiene sentido el discurso acerca de la verdad de las interpretaciones. En este sentido, el debate en términos de corrección es más abarcativo. Véase Canale (2012) y Ruiz Manero (2012).

${ }^{40} \mathrm{Creo}$, en cambio, que es importante tener presente que esta es una cuestión contingente, que depende de cada sistema jurídico.
} 
enfatizan el rol que el derecho desempeña en general en nuestras vidas, y el carácter residual de los conflictos interpretativos si tenemos en cuenta tal incidencia. Pero entonces, si el derecho tiene un impacto en casi todo lo que hacemos, y solo algunos casos llegan a los tribunales, y además solo algunos de ellos plantean problemas interpretativos, enfatizar que siempre hay diferentes instrumentos y que el juez elige supondría presentar una imagen distorsionada de la práctica jurídica.

El realismo moderado podría, por último, rechazar el discurso acerca de la corrección y enfatizar que los jueces simplemente buscan alcanzar ciertos objetivos o evitar consecuencias indeseables. Pero además de obviar rasgos prominentes de la práctica jurídica, ello no le permitiría diferenciarse de la pesadilla ${ }^{41}$.

Cabría cuestionar si el discurso respecto de la corrección de las interpretaciones es sustancialmente distinto del de la verdad de los enunciados interpretativos. Si bien es posible que toda posición interpretativa en términos de corrección sea reconducible, en última instancia, a un discurso acerca de la verdad de los enunciados interpretativos, creo que resulta preferible hacer meramente referencia a la corrección de las interpretaciones. Con ello me refiero a que el elemento central se relaciona con el reconocimiento (o no) de errores en las interpretaciones. Como ya he señalado, creo que ello es conveniente debido a que el discurso acerca de la verdad de los enunciados interpretativos es poco frecuente, no solo a nivel teórico sino también entre los propios participantes. Además, no existe un gran consenso acerca de la naturaleza de los enunciados interpretativos, ni acerca de cómo cabe entender la verdad en este ámbito. El debate acerca de la corrección es por ello más abarcativo, y permite clasificar las distintas posiciones con independencia de aspectos tan controvertidos como la posición que los autores asumen respecto de la verdad de los enunciados. Y es también un discurso mucho más frecuente entre participantes y teóricos. Finalmente, la posibilidad de predicar error sí constituye un aspecto central en el debate de la interpretación jurídica, por lo que permite una mejor comprensión de las distintas posiciones. Obviamente, hacer referencia a la corrección también requiere de mayores precisiones, y este trabajo ha sido solo una primera aproximación, muy tentativa, a la cuestión. Pero en todo caso considero, y este ha sido mi propósito central, que debe prescindirse de la verdad de los enunciados interpretativos para avanzar en el debate acerca de la interpretación jurídica.

\section{BIBLIOGRAFÍA}

Arena, F. (2013). Una alternativa para el escepticismo interpretativo: convenciones y cuasirrealismo en la interpretación jurídica, pendiente de publicación.

Barberis, M. (2000). Lo scetticismo imaginario. Nove obiezioni agli scettici à la génoise, "Analisi e Diritto", 1-36.

${ }^{41}$ Guastini, no obstante, podría insistir en que se diferencia de la pesadilla porque entiende que en determinados casos el juez crea, en lugar de interpretar. Como ya he señalado con anterioridad, la diferencia es entonces meramente terminológica, y además no está claro qué se gana enfatizando ese punto si la consecuencia parece ser la misma: el juez emplea un instrumento no idóneo para desempeñar su labor interpretativa. 
Canale, D. (2012). “Teorías de la interpretación jurídica y teorías del significado”, "Discusiones", XI, 135-165.

Chiassoni, P. (1998). L'ineluttabile scetticismo della "scuola genovese", "Analisi e Diritto", 21-76.

DuARTE D'Almeida, L. (2011). Legal Statements and normative language, "Law and Philosophy", 30, 167-199.

FERrer Beltrán, J. (2010). Sobre la posibilidad del error judicial y los desacuerdos irrecusables en el derecho. Once comentarios a las tesis de José Juan Moreso, in Moreso, J., Prieto Sanchís, L., Ferrer Beltrán, J., Los desacuerdos en el derecho, Madrid, Fundación coloquio jurídico europeo, 147-182.

Ferrer, J., Ratti, G. (eds.) (2011). El realismo jurídico genovés, Barcelona, Marcial Pons.

Guastini, R. (1999). Distinguiendo. Estudios de teoría y metateoría del derecho, Barcelona, Gedisa.

GuAstini, R. (2008). Estudios sobre la interpretación jurídica, $8^{\mathrm{a}}$ ed, México, DF, Editorial Porrúa.

Guastini, R. (2010). Nuevos estudios sobre la interpretación, Bogotá, Universidad Externado de Colombia.

Guastini, R. (2012). El escepticismo ante las reglas replanteado, "Discusiones", XI, 27-57.

Hart, H. (1980). El nuevo desafío del positivismo jurídico, "Sistema", 36, 3-18.

Hart, H. (1982). Essays on Bentham, Oxford, Clarendon Press.

Hart, H. (1994). The Concept of Law, $2^{\mathrm{a}}$ ed, Oxford, Oxford University Press.

HERNÁNDEZ Marín, R. (2008). Sobre ontología jurídica e interpretación del derecho, "Isonomía", 29, 33-78.

Holton, R. (1998). Positivism and the internal point of view, "Law and Philosophy", 17, 597-625.

KRIPKe, S. (1980). Naming and necessity, Cambridge, Harvard University Press.

KRIPKE, S. (1982). Wittgenstein on rules and private language, Oxford, Basil Blackwell.

Lifante Vidal, I. (2012). Distinciones y paralogismos. A propósito del escepticismo guastiniano, "Discusiones", X1, 59-85.

Moreso, J.J. (1997). La indeterminación del derecho y la interpretación de la Constitución, Madrid, Centro de Estudios Políticos y Constitucionales.

Muffato, N. (2013). Tertium non datur: Sulla pretesa autonomia concettuale degli asserti distaccati, en prensa.

Papayannis, D. (2013). El aspecto interpretativo de la regla de reconocimiento, manuscrito inédito.

Putnam, H. (1975). Mind, Language and Reality: Philosophical Papers, vol. 2, Cambridge, Cambridge University Press.

Ramírez Ludeña, L. (2012). El desvelo de la pesadilla. Una respuesta a "El escepticismo ante las reglas replanteado", "Discusiones", XI, 87-116.

Raz, J. (1980). The Authority of Law, $2^{\mathrm{a}}$ ed, Oxford, Oxford University Press.

RAz, J. (1980), The Concept of a Legal System, $2^{\mathrm{a}}$ ed, Oxford: Clarendon Press.

Redondo, C. (1999), Reasons for action and the law, Dordrecht, Kluwer.

Ruiz Manero, J. (2012). Epílogo: interpretación jurídica y direcciones de ajuste, "Discusiones", XI, 203-219.

Shapiro, S. (2011). Legality, Cambridge, Harvard University Press.

Tarello, G., (1974). La semántica del néustico. Observaciones sobre la "parte descriptiva" de los enunciados prescriptivos, tr. esp. in Ferrer, J., Ratti, G. (eds.) (2011), El realismo jurídico genovés, Barcelona, Marcial Pons, 15-39.

Тон, K. (2007). Raz on detachement, acceptance and describability, "Oxford Journal of Legal Studies", 27, 403-427. 
